\title{
Selective radiosensitization by nitazoxanide of quiescent clonogenic colon cancer tumour cells
}

\author{
HENNING KARLSSON $^{1}$, MÅRTEN FRYKNÄS ${ }^{1}$, WOJCIECH SENKOWSKI ${ }^{1}$, \\ ROLF LARSSON $^{1}$ and PETER NYGREN ${ }^{2}$
}

Departments of ${ }^{1}$ Medical Sciences and ${ }^{2}$ Immunology, Genetics and Pathology, Uppsala University, Uppsala S-751 85, Sweden

Received May 24, 2021; Accepted January 31, 2022

DOI: $10.3892 / \mathrm{ol} .2022 .13243$

\begin{abstract}
Nitazoxanide is a Food and Drug Administrationapproved antiprotozoal drug recently demonstrated to be selectively active against quiescent and glucose-deprived tumour cells. This drug also has several characteristics that suggest its potential as a radiosensitizer. The present study aimed to investigate the interaction between nitazoxanide and radiation on human colon cancer cells cultured as monolayers, and to mimic key features of solid tumours in patients, as spheroids, as well as in xenografts in mice. In the present study, colon cancer HCT116 green fluorescent protein (GFP) cells were exposed to nitazoxanide, radiation or their combination. Cell survival was analysed by using total cell kill and clonogenic assays. DNA double-strand breaks were evaluated in the spheroid experiments, and HCT116 GFP cell xenograft tumours in mice were used to investigate the effect of nitazoxanide and radiation in vivo. In the clonogenic assay, nitazoxanide synergistically and selectively sensitized cells grown as spheroids to radiation. However, this was not observed in cells cultured as monolayers, as demonstrated in the total cell kill assays, and much less with the clinically established sensitizer 5-fluorouracil. The sensitizing effect from nitazoxanide was confirmed via spheroid $\gamma$-H2A histone family member $\mathrm{X}$ staining. Nitazoxanide and radiation alone similarly inhibited the growth of HCT116 GFP cell xenograft tumours in mice with no evidence of synergistic interaction. In conclusion, nitazoxanide selectively targeted quiescent glucose-deprived tumour cells and sensitized these cells to radiation in vitro. Nitazoxanide also inhibited tumour growth in vivo. Thus, nitazoxanide is a candidate for repurposing into an anticancer drug, including its use as a radiosensitizer.
\end{abstract}

Correspondence to: Dr Henning Karlsson, Department of Medical Sciences, Uppsala University, Entrance 61, Sjukhusvägen 9, Uppsala S-751 85, Sweden

E-mail: henning.karlsson@medsci.uu.se

Key words: nitazoxanide, spheroid, 3D, radiosensitizer, clonogenic

\section{Introduction}

Although cytotoxic drugs, such as 5-fluorouracil (5-FU), platinums, taxanes and mitomycin, as well as some of the new targeted drugs, including the EGFR-antibody cetuximab, have been successfully used together with radiotherapy, it is not always apparent whether chemoradiotherapy provides a benefit over radiotherapy alone (1-6). Thus, there is a requirement for drugs that more selectively kill and/or radiosensitize tumour cells that are resistant to chemotherapy and radiotherapy.

The dominant mode of action of photon radiation used in radiotherapy for cancer is indirect radiolysis of water molecules, which generates free oxygen radicals. This will induce both DNA-DNA and DNA-protein cross-links and base lesions, as well as cause damage to sugar moieties in the backbone of DNA, resulting in single-strand breaks and double-strand breaks (DSBs). The number of DSBs is considered to be the major determinant of radiation-induced damage. Increased oxygen levels cause tumours to be more sensitive to radiation and, although both normal and tumour tissues vary in oxygenation, only tumours are considered to be sufficiently hypoxic to impair cell killing induced by radiation (7-11).

Nitazoxanide is a Food and Drug Administration-approved antiprotozoal drug with several characteristics theoretically suitable for the radiosensitization of solid tumours (12). This drug was recently identified to be selectively toxic to quiescent and glucose-deprived tumour cells and was described as an oxidative phosphorylation (OXPHOS) inhibitor (12). Nitazoxanide was also shown to reduce hypoxia (12) in spheroids and it is well established that hypoxic areas in tumours are associated with resistance to radiotherapy (13). These findings suggest that nitazoxanide, by increasing tumour oxygenation, could potentially enhance the number of DSBs caused by the radiation-induced formation of free oxygen radicals in hypoxic tumours.

Based on the aforementioned findings, the present study aimed to investigate a potential radiosensitization effect of nitazoxanide in clinically relevant models that have been recently described (12). To evaluate the effect of nitazoxanide and radiation, the colon cancer cell line, HCT116 green fluorescent protein (GFP), was cultured as monolayers or spheroids and evaluated using total cell kill or clonogenic assays as read-outs. The expression level of $\gamma$-H2A histone family member X (H2AX) was used to evaluate DSBs and the 
in vivo antitumour effect was evaluated using HCT116 GFP cell xenograft tumours in mice.

\section{Materials and methods}

Cells and cell culture. HCT116 GFP cells (human colon cancer cell line HCT116 transfected with GFP; AntiCancer, Inc.) were cultured at $37^{\circ} \mathrm{C}$ in a humidified incubator containing $5 \% \mathrm{CO}_{2}$ in McCoy's $5 \mathrm{~A}$ medium (Sigma-Aldrich; Merck KGaA) supplemented with $10 \%$ heat-inactivated FBS, L-glutamine $(2 \mathrm{mM})$, penicillin $(100 \mathrm{U} / \mathrm{ml})$ and streptomycin $(100 \mu \mathrm{g} / \mathrm{ml})$. The morphology and proliferation of cells were monitored on a weekly basis and the cell line was split twice a week. The cell line was authenticated via short-term repeat analysis performed by the cell bank (AntiCancer, Inc.). Cells were passaged for $<3$ months after resuscitation.

\section{Drugs, irradiation and cell culture experiments}

Drugs. Nitazoxanide was obtained from Selleck Chemicals. For comparison and reference, the standard cytotoxic drug 5-FU (Sigma-Aldrich; Merck KGaA) was included. 5-FU is the backbone therapy for colorectal cancer chemotherapy regimens and is also in clinical use as a radiosensitizer $(1,14)$.

The oxygen and moisture free MiniPod ${ }^{\mathrm{TM}}$ system (Roylan Developments Ltd.) was used to store source plates that were prepared with drugs solubilized in DMSO. The liquid handling system $\mathrm{ECHO}^{\circledR} 550$ (Labcyte, Inc.) was used to add the drugs to the experimental plates.

Monolayer experiments. The monolayer experiments were performed as described previously, with minor modifications (15). Briefly, on day 0 of the experiment, $50 \mu \mathrm{l}$ cell suspension (1,000 cells/well) was added into 384-well plates and pre-incubated in $37^{\circ} \mathrm{C}$ overnight at standard cell culture conditions. On day 1 , the drug was added and experimental plates were irradiated with an external low dose-rate $\gamma$ radiation source (GammaCell 40 Exactor; Best Theratronics, Ltd.) 4-6 h thereafter. Cell viability was assessed using the fluorometric microculture cytotoxicity assay (FMCA) and clonogenic assay as later described.

Spheroid experiments. The formation of HCT116 GFP quiescent multicellular tumour spheroids (Q-MCTS) was previously described in detail (12). Briefly, 384-well Corning ${ }^{\circledR}$ black clear bottom ultra-low attachment microplates (Corning, Inc.) were used with $50 \mu 1$ cell suspension, with 10,000 cells seeded into each well on day 0 . Q-MCTS formed during the 7 day culture without a change of medium. The $\mathrm{ECHO}^{\circledR} 550$ liquid handler was used to add the drugs on day 7. Spheroids were incubated with drugs for 4-6 $\mathrm{h}$ and then irradiated as aforementioned. Following 7 days of drug incubation, cell viability was assessed using the FMCA and the GFP assay for total cell kill, while a clonogenic assay was used for the assessment of the effect in clonogenic cells only (see below).

\section{Measurement of cellular cytotoxicity}

Total cell kill assay in monolayers. Following 3 or 7 days of drug incubation, cytotoxicity in the total cell population was assessed using FMCA as previously described (15). The FMCA is based on fluorescein diacetate that is converted to fluorescent fluorescein by viable cells with an intact plasma membrane (15).

Total cell kill assay in spheroids. Following the aforementioned spheroid experiments, cytotoxicity in the total cell population was assessed using FMCA and GFP assays. The GFP assay is based on the fluorescent signal generated from viable HCT116 GFP cells of the intact spheroids, as described previously (12). In the FMCA, $40 \mu \mathrm{l}$ medium was aspirated from each well and $50 \mu \mathrm{l}$ Accumax/well was added. The plates were then incubated at $37^{\circ} \mathrm{C}$ for $30 \mathrm{~min}$, followed by the dissociation of spheroids into single cells using a multipipette. The FMCA procedure was then performed for the monolayer cultured cells, as described previously (15).

Clonogenic assay on monolayer and spheroid cultures. In the monolayer cultures, $\sim 20 \mathrm{~h}$ after radiation, $40 \mu \mathrm{l}$ medium was aspirated from each well and $50 \mu 1$ Accumax/well was added. After incubation at $37^{\circ} \mathrm{C}$ for $30 \mathrm{~min}$, mixing, centrifugation, removal of Accumax solution, the addition of $50 \mu \mathrm{l}$ fresh medium and subsequent mixing, $20 \mu \mathrm{l}$ cell solution from each well was transferred together with $3 \mathrm{ml}$ fresh medium into each well in 6-well plates (Nalge Nunc International; Thermo Fisher Scientific, Inc.).

Spheroids were dissociated into single cells as aforementioned at $\sim 20 \mathrm{~h}$ after radiation, followed by removal of Accumax solution and then addition of $50 \mu 1$ fresh medium. In total, $20 \mu \mathrm{l}$ cell solution from each well was transferred together with $3 \mathrm{ml}$ fresh medium to 6-well plates (Nalge Nunc International; Thermo Fisher Scientific, Inc.) and the procedure was then performed identical to that described for monolayer experiments.

The 6-well plates were incubated at $37^{\circ} \mathrm{C}$ for 10 days and cells were then fixed and stained as previously described by Franken et al (16). A Canon iR-ADV C5235i printer (Canon, Inc.) was used to scan plates and number of colonies were then counted on the computer screen. Only colonies with $>50$ cells were counted.

Assessment of DSBs via immunohistochemistry (IHC). The formation of spheroids and exposure to drugs and radiation were performed as previously described. Subsequently, $24 \mathrm{~h}$ after radiation, spheroids were harvested into Eppendorf tubes and washed once in PBS. Spheroids were embedded in paraffin, sectioned and evaluated for DSBs using the anti- $\gamma \mathrm{H} 2 \mathrm{AX}$ (phosphorylated S139) antibody [9F3] against the synthetic peptide phosphorylated (Ser139) human H2AX (cat. no. ab26350; Abcam) and for apoptosis using antibodies against Annexin V (cat. no. ab108321; Abcam) and caspase-3 (cat. no. 9664; Cell Signaling), according to the manufacturer's protocols. In the staining process, the commercially available MACH1 Universal HRP-Polymer Detection kit (Biocare Medical) with peroxide block for 5 min with Biocare's Peroxidazed 1 (without protein block) was used. Antibodies were used at the following dilutions: H2AX (1:1,000), Annexin V (1:1,000) and caspase-3 (1:100). The incubation time was $20 \mathrm{~min}$. IHC staining was assessed using a light microscope at $\mathrm{x} 400$ magnification.

Assessment of nitazoxanide and radiation effects in tumour xenografts. In total, 40 female 8-week old NMRI nu/nu mice 
Table I. Interaction ratios of nitazoxanide and radiation combinations in the FMCA assay.

\begin{tabular}{|c|c|c|c|c|c|c|c|}
\hline \multirow[b]{2}{*}{$\mathrm{SI}_{\mathrm{o}} / \mathrm{SI}_{\mathrm{e}}(\mu \mathrm{M})$} & \multicolumn{3}{|c|}{ Monolayer (3 days) } & \multicolumn{3}{|c|}{ Monolayer (7 days) } & \multirow{2}{*}{$\begin{array}{c}\text { Spheroids } \\
6 \mathrm{~Gy}\end{array}$} \\
\hline & 2 Gy & 4 Gy & $6 \mathrm{~Gy}$ & 2 Gy & 4 Gy & $6 \mathrm{~Gy}$ & \\
\hline 100 & - & - & - & - & - & - & - \\
\hline 50 & - & - & - & - & - & - & - \\
\hline 25 & 1.030 & 1.154 & 1.390 & $1.221^{\mathrm{a}}$ & $1.156^{\mathrm{a}}$ & $1.263^{\mathrm{a}}$ & - \\
\hline 12.5 & 1.096 & 1.123 & $1.269^{\mathrm{a}}$ & $2.186^{\mathrm{a}}$ & $3.742^{\mathrm{a}}$ & $4.435^{\mathrm{a}}$ & 1.043 \\
\hline 6.5 & 1.064 & 1.091 & 1.169 & $1.301^{\mathrm{a}}$ & $2.544^{\mathrm{a}}$ & $3.254^{\mathrm{a}}$ & $0.996^{\mathrm{b}}$ \\
\hline 3 & 1.060 & $1.063^{\mathrm{a}}$ & 1.152 & 1.201 & $1.552^{\mathrm{a}}$ & $1.819^{\mathrm{a}}$ & 1.037 \\
\hline 1.5 & 1.105 & 1.096 & 1.174 & 1.051 & $1.267^{\mathrm{a}}$ & $1.379^{\mathrm{a}}$ & 1.020 \\
\hline 1 & $1.047^{\mathrm{a}}$ & 1.042 & 1.144 & 1.057 & 1.195 & $1.381^{\mathrm{a}}$ & 1.054 \\
\hline 0.5 & 1.032 & 1.035 & 1.094 & 1.023 & $1.139^{\mathrm{a}}$ & 1.292 & 1.064 \\
\hline
\end{tabular}

${ }^{\mathrm{a}} \mathrm{P}<0.05 .{ }^{\mathrm{b}} \mathrm{SI}_{\mathrm{o}} / \mathrm{SI}_{\mathrm{e}}$ ratios $<1$ are considered to indicate synergy. Ratios $>1$ indicate subadditive or antagonistic interactions. A two-tailed $\mathrm{P}<0.05$ was used to indicate interaction ratios significantly different from 1. Data are presented as the mean interaction ratio $\left(\mathrm{SI}_{\mathrm{o}} / \mathrm{SI}_{\mathrm{e}}\right)$ for three or seven individual experiments for monolayers and spheroids, respectively. Duplicate wells were used for each drug concentration. A one-sample t-test was used to calculate interaction ratios different from 1. -, not tested due to $\mathrm{SI}_{\mathrm{d}}<25 \%$.

(Crl:NMRI-Foxn1nu) from Charles River Laboratories were injected subcutaneously at the right flank with a 50- $\mu$ l cell suspension containing $5 \times 10^{6}$ HCT116 GFP cells mixed with $50 \mu 1$ Matrigel. The animals were included into the study in groups of 10 animals/group when the majority of the tumours had reached a size of $\sim 0.1 \mathrm{~cm}^{3}$. The groups were as follows: Group 1, vehicle; group 2, vehicle + irradiation; group 3, nitazoxanide; and group 4, nitazoxanide + irradiation. Starting at day 0 , drug/vehicle administration occurred twice daily for 3 days (there was only one administration on day 2). Vehicle control (1\% carboxymethylcellulose in PBS with $8 \%$ DMSO) and nitazoxanide $(200 \mathrm{mg} / \mathrm{kg})$ were administered via oral gavage. Temperature was kept at $21-22^{\circ} \mathrm{C}$, humidity was $50-60 \%$ and a 12-h light, 12-h dark cycle was applied. Autoclaved tap water was available ad libitum in water bottles and the animals received $\mathrm{R} 70$, irradiated, diet (Lantmännen $\mathrm{AB}$ ).

At $\sim 4 \mathrm{~h}$ after the last drug administration, the tumours of the animals were irradiated with $6 \mathrm{~Gy}$. The irradiated area, with the tumour centred, measured $2 \times 2 \mathrm{~cm}$ on the right rear flank. With the setting of $320 \mathrm{kV}$ and filter F2, the total radiation dose of $6 \mathrm{~Gy}$ was delivered at $0.73 \mathrm{~Gy} / \mathrm{min}$. An X-RAD 340 (PXi; Precision X-ray, Inc.) was used for the radiation. The length and width of each tumour was measured using a calliper twice weekly, and the tumour volume (TV) was calculated using the formula: Length $(\mathrm{cm}) \mathrm{x}$ width $(\mathrm{cm})$ $\mathrm{x}$ width $(\mathrm{cm}) \mathrm{x} 0.44$. Body weight was recorded at the start of the study, along with tumour measurements, and at the end of the study. The animals were checked daily for any change in activity and appearance, as signs of a change in general health status. The animals were euthanized via cervical dislocation at the end of the study on day 28 , and the tumours were then dissected and placed into $4 \%$ buffered formaldehyde solution for subsequent histopathological examination.

Tumours were embedded in paraffin, sectioned, evaluated for H\&E staining and electronically scanned according to standard protocols. The analysis algorithm 'Positive Pixel Count v9' in Aperio ImageScope (v12.3.2.8013; Leica Microsystems
$\mathrm{GmbH}$ ) was slightly modified (Table SI). Using this algorithm, strong positive areas (SPA) corresponded well to areas with cells visually classified as HCT116 GFP cells under the microscope. Thus, SPA/(weak positive areas (WPA) + SPA) x100 was used as a surrogate marker for the fraction of HCT116 GFP cells in the tumour and was referred to as the tumour cell percentage (TCP). The tumour cell volume (TCV) was calculated as TCP x TV/100.

Statistical analysis and presentation. The calculations and graphical presentations were performed in GraphPad Prism 7 (GraphPad Software, Inc.). Dose-response curves are presented as the mean \pm SEM for the number of experiments indicated. Cell viability data in the FMCA for both spheroid and monolayer experiments are presented as survival index (SI), defined as the fluorescence in experimental wells in \% of that in unexposed control wells, with the fluorescence of the blank wells subtracted. An AUTO SI was calculated in the GFP assay, and defined as the spheroid fluorescence in experimental wells in $\%$ of that in the same wells immediately before addition of drug/radiation 7 days earlier.

In the clonogenic assays, a slightly modified definition was used to calculate surviving fraction (SF), which was defined as the number of colonies in $\%$ of that in unexposed control wells, since plating efficiency was not assessed. The interaction between drug and radiation was characterized using the mean SI (or SF) for wells treated with drug only $\left(\mathrm{SI}_{d}\right.$ or $\mathrm{SF}_{\mathrm{d}}$ ) and the mean SI (or SF) for wells irradiated only $\left(\mathrm{SI}_{\mathrm{r}}\right.$ or $\mathrm{SF}_{\mathrm{r}}$ ). An expected combination $\mathrm{SI}$ or $\mathrm{SF}\left(\mathrm{SI}_{\mathrm{e}}\right.$ or $\left.\mathrm{SF}_{\mathrm{e}}\right)$ was calculated as follows: $\mathrm{SI}_{d} \times \mathrm{SI}_{\mathrm{r}}=\mathrm{SI}_{\mathrm{e}}\left(\right.$ or $\mathrm{SF}_{\mathrm{d}} \times \mathrm{SF}_{\mathrm{r}}=\mathrm{SF}_{\mathrm{e}}$ ), in accordance with independent Bliss interaction (17). To obtain an interaction ratio for each individual experiment, the SI (or SF) actually observed for the combination $\mathrm{SI}_{\mathrm{o}}$ (or $\mathrm{SF}_{\mathrm{o}}$ ) was divided by $\mathrm{SI}_{\mathrm{e}}$ (or $\mathrm{SF}_{\mathrm{e}}$ ). For each group in Tables I and II, based on the number of experiments indicated in the table legend, one-sample t-test was used to calculate if the interaction ratio was different from the value 1 . 
Table II. Interaction ratios of drug and radiation combinations in the clonogenic assay in cells cultured as spheroids.

\begin{tabular}{lcccc}
\hline $\mathrm{SF}_{\mathrm{o}} / \mathrm{SF}_{\mathrm{e}}(\mu \mathrm{M})$ & 5 -FU + 4 Gy & $5-\mathrm{FU}+6 \mathrm{~Gy}$ & Nitazoxanide + 4 Gy & Nitazoxanide $+6 \mathrm{~Gy}$ \\
\hline 100 & $0.57^{\mathrm{b}}$ & 0.67 & - & - \\
50 & $0.61^{\mathrm{a}}$ & 0.58 & - & - \\
12.5 & 0.88 & 0.96 & - & - \\
6.5 & 0.81 & 0.90 & $0.16^{\mathrm{b}}$ & $0.14^{\mathrm{b}}$ \\
3 & - & - & $0.47^{\mathrm{c}}$ & $0.39^{\mathrm{a}}$ \\
\hline
\end{tabular}

${ }^{\mathrm{a}} \mathrm{P}<0.05,{ }^{\mathrm{b}} \mathrm{P}<0.01$ and ${ }^{\mathrm{c}} \mathrm{P}=0.0682$. Data are presented as the mean interaction ratio $\left(\mathrm{SF}_{\mathrm{o}} / \mathrm{SF}_{\mathrm{e}}\right)$ for $2-3$ individual experiments with triplicate wells for each drug concentration. One-sample t-test was used to calculate interaction ratios different from 1 . All available data had an $\mathrm{SF}_{\mathrm{o}} / \mathrm{SF}_{\mathrm{e}}$ ratio $<1$, which was considered to indicate synergy. A two-tailed $\mathrm{P}<0.05$ was used to indicate interaction ratios significantly different from $1 .-$, not tested.

In the tumour xenograft experiments, TVs are presented as the mean \pm SEM. Differences in TV between the groups were calculated using a repeated measures (from day 0 to 28) two-way ANOVA followed by Tukey's multiple comparisons test in GraphPad Prism. When animals were euthanized pre-term, the last data point was carried forward and included in the calculations of the means and in the statistical analysis. Differences in TCP and TCV were calculated using one-way ANOVA followed by Tukey's multiple comparisons test in GraphPad Prism. In total, three animals that were euthanized pre-term were excluded from the analysis. $\mathrm{P}<0.05$ was considered to indicate a statistically significant difference.

\section{Results}

Total cell kill assay in monolayer-and spheroid cultures. The selective activity of nitazoxanide against nutrient-deprived cells grown as multicellular tumour spheroids $\left(\mathrm{IC}_{50}\right.$ 1.44 and $2.41 \mu \mathrm{M}$ in the GFP assay and FMCA, respectively), compared with monolayer cultures $\left(\mathrm{IC}_{50} 5.35 \mu \mathrm{M}\right.$ in the FMCA), following 7 days of drug exposure was confirmed in the total cell kill assay (Fig. 1A upper panel and B). This effect was in contrast to most other drugs, which are usually considerably more active against monolayer cells compared with cells grown as spheroids (12). Radiation alone was modestly active in monolayer cultures, with a slightly greater effect after 3 vs. 7 days of culture following radiation (Fig. 1A lower panel).

Interestingly, in the monolayer experiments, radiation seemingly 'protected' the cells from the effect of nitazoxanide after 7 days, but not after 3 days, of drug exposure (Fig. 1A upper panel). Cell survival was higher after 7 days compared with that at 3 days after radiation only, indicating that the radiation doses used in this experiment induced transient inhibition of cell proliferation rather than cell death.

Radiation at 6 Gy had little effect on cell survival in spheroids (SI 92 and 93\% in the GFP assay and FMCA, respectively). Thus, monolayer cells were more sensitive than spheroids to radiation (Fig. 1A and B). Moreover, nitazoxanide showed no statistically significant synergistic effects with radiation in the total cell kill assays for monolayer and spheroid cultures (Table I).

Clonogenic assay in monolayer and spheroid cultures. The nitazoxanide-radiation interaction was furtherinvestigated in the clonogenic assay (Fig. 1C-F; Table II). Nitazoxanide interacted synergistically with radiation in a radiation dose-dependent manner in cells grown as spheroids (Fig. 1E and F; Table II). By contrast, there was no synergy in monolayer cell cultures (Fig. 1C and D). The clonogenicity of cells from spheroids was markedly affected by radiation only [SF 36.1 \pm 5.16 and $8.13 \pm 1.94 \%$ (mean \pm SEM) at 4 and 6 Gy, respectively], but less so than cells from monolayers [SF $40.3 \pm 6.05$ and $10.1 \pm 2.57 \%$ (mean \pm SEM) at 2 and 4 Gy, respectively]. Although the clinically most used radiosensitizer in colorectal cancer, 5-FU, at high concentrations $(50-100 \mu \mathrm{M})$ showed radiosensitizing effects in spheroids, the effect was not as strong as that with nitazoxanide (Table II). The lack of the effect of nitazoxanide alone in the clonogenic assay in spheroids was expected from the short incubation time in this assay.

IHC for assessment of DSBs. A necrosis-like pattern was observed centrally in spheroids exposed to nitazoxanide (Fig. 2). Since $\gamma$-H2AX expression may reflect apoptosis, as well as DSBs, apoptosis was evaluated using antibodies against both Annexin V and caspase-3 (Figs. S1 and S2) No notable difference was observed between the treatment groups and, thus, a difference in $\gamma$-H2AX expression, as determined via IHC, was considered due to DSBs.

DSBs were induced in spheroids by 3 and $6.5 \mu \mathrm{M}$ nitazoxanide, and were determined using the IHC assessment of $\gamma-\mathrm{H} 2 \mathrm{AX}$ expression. Exposure to nitazoxanide and radiation resulted in higher $\gamma-\mathrm{H} 2 \mathrm{AX}$ expression compared with that after each treatment alone (Fig. 2). The intensity of the $\gamma-\mathrm{H} 2 \mathrm{AX}$ staining was considerably stronger in central parts of spheroids exposed to the combination compared with spheroids exposed to nitazoxanide or radiation only, which was indicative of a selective radiosensitizing effect towards more centrally located cells. As shown in Fig. 2, DSBs were markedly induced in spheroids by $6 \mathrm{~Gy}$ of radiation.

Nitazoxanide and radiation effects in tumour xenografts. The antitumour activity and radiosensitizing properties of nitazoxanide were investigated in HCT116 GFP cell xenograft tumours in mice. It was found that, over time, ulcers developed on some tumours that penetrated the skin, and one animal in each group, but the nitazoxanide + radiation group, was euthanized pre-term. There was a temporary decrease in body weight after the treatments started in the two irradiated groups, but the decrease was recaptured 4 days later, and 

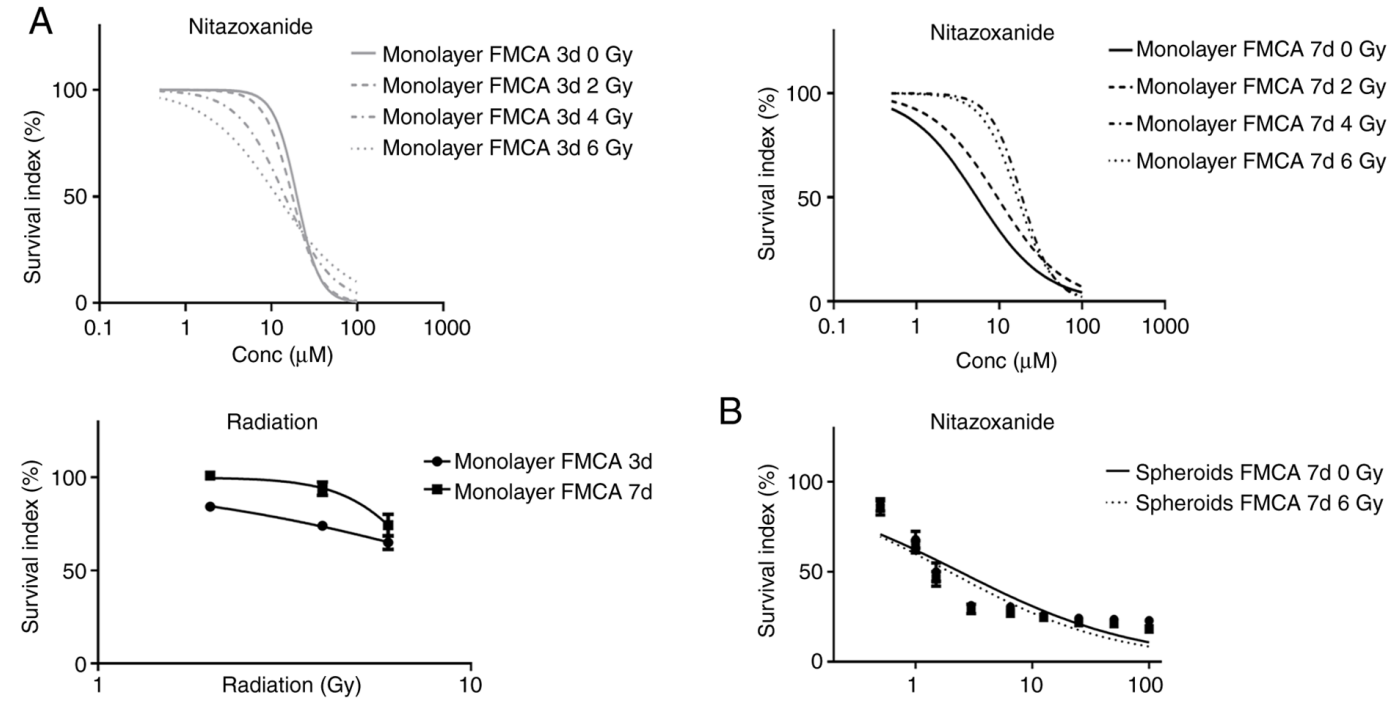

B
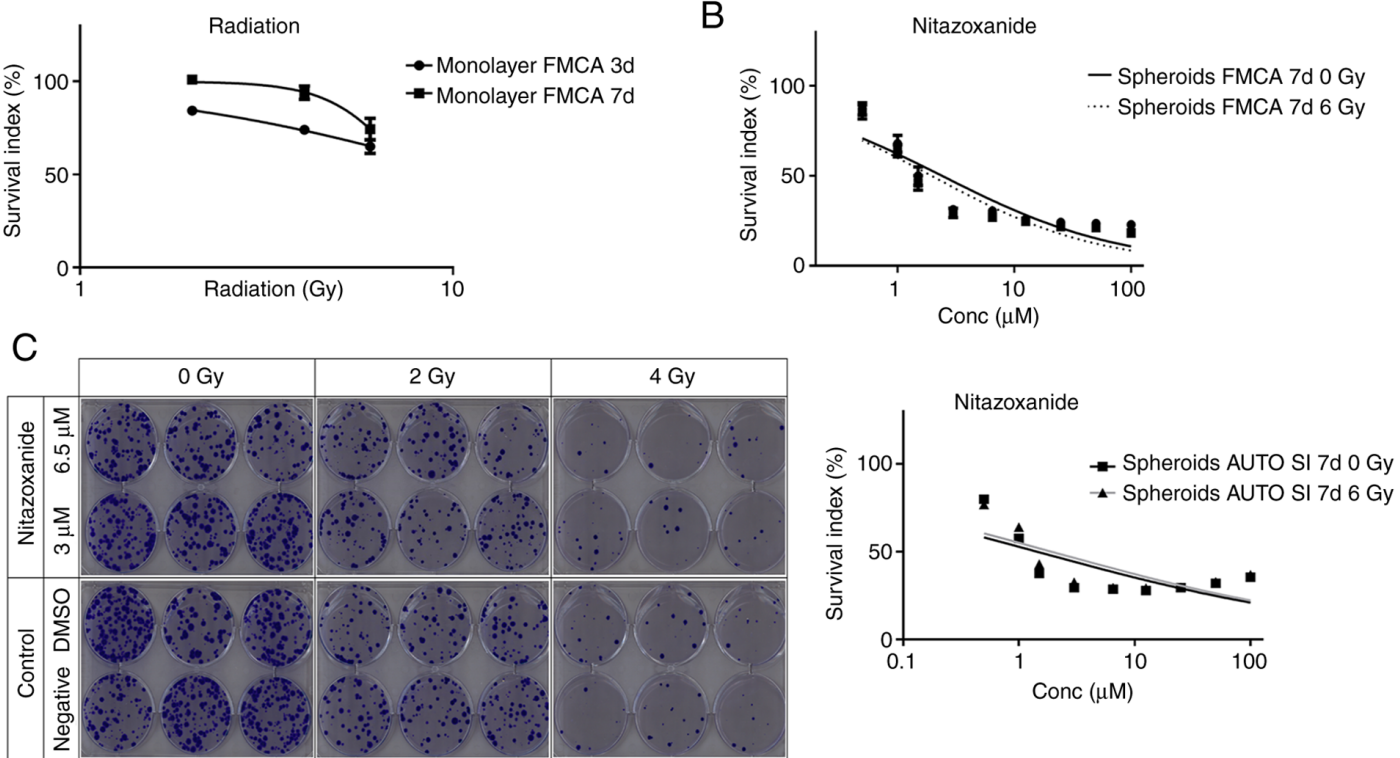

D

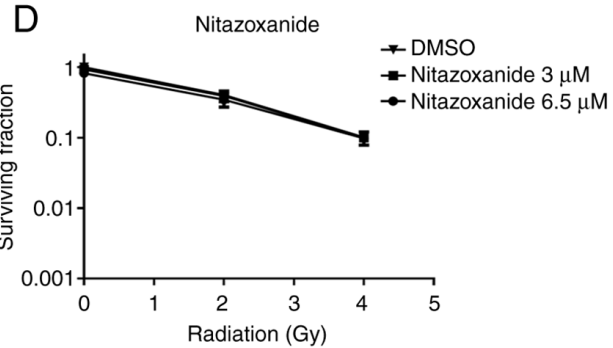

$\mathrm{F}$

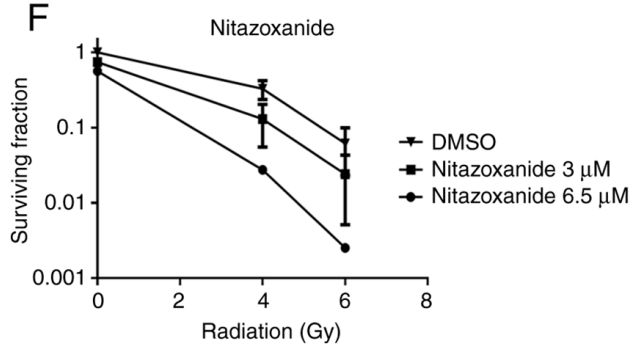

E

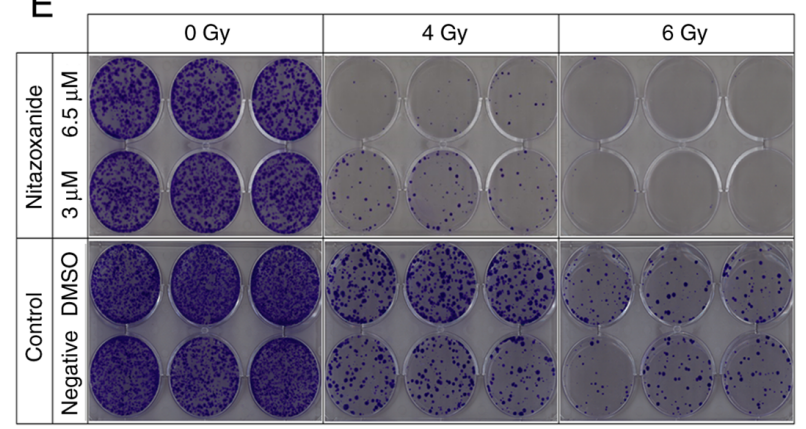

Figure 1. Cell survival in the FMCA-, GFP- and clonogenic assays. (A) Cell survival in the FMCA assay, expressed as SI of HCT116 GFP cells cultured as monolayers, pre-incubated overnight and then incubated with drugs for 3 or 7 days with irradiation (2-6 Gy) at 4-6 h after addition of drug (upper panel). Effect of 2, 4 and 6 Gy radiation in HCT116 GFP cells incubated with DMSO for 3 and 7 days (lower panel). n=3 independent experiments; SEM (generally <8) were omitted in upper panels. (B) Cell survival in the total cell kill assay, expressed as SI (FMCA, upper panel) or AUTO SI (GFP assay, lower panel) of HCT116 GFP cells cultured as spheroids for 7 days, then incubated with drugs for 7 days with irradiation (6 Gy) $4-6 \mathrm{~h}$ after addition of drug. $\mathrm{n}=7-8$ independent experiments. Drug concentrations used were: $0.5,1.0,1.5,3.0,6.5,12.5,25,50$ and $100 \mu \mathrm{M}$. (C) Clonogenic assay with nitazoxanide, shown as growth of HCT116 GFP cells cultured as monolayers, pre-incubated overnight in 384-well plates, with no irradiation (control) or irradiated (2 or 4 Gy) at 4-6 h after drug addition and $20 \mathrm{~h}$ later dissociated into single cells, transferred to six-well plates and incubated for 10 days. Triplicate wells were used for each drug concentration. (D) Cell survival in the clonogenic assay, expressed as surviving fraction of HCT116 GFP cells cultured as monolayers, pre-incubated overnight in 384-well plates, with no irradiation (DMSO control) or irradiated ( 2 or $4 \mathrm{~Gy}$ ) at $4-6 \mathrm{~h}$ after drug addition and $20 \mathrm{~h}$ later dissociated into single cells, transferred to six-well plates and incubated for 10 days. $n=4$ independent experiments. (E) Clonogenic assay with nitazoxanide, shown as growth of HCT116 GFP cells cultured as spheroids for 7 days, with no irradiation (control) or irradiated (4 or $6 \mathrm{~Gy}$ ) at $4-6 \mathrm{~h}$ after drug addition and $20 \mathrm{~h}$ later dissociated into single cells, transferred to six-well plates and incubated for 10 days. Triplicate wells were used for each drug concentration. (F) Cell survival in the clonogenic assay, expressed as surviving fraction of HCT116 GFP cells cultured as spheroids for 7, with no irradiation (DMSO control) or irradiated (4 or $6 \mathrm{~Gy}$ ) at 4-6 h after drug addition, and $20 \mathrm{~h}$ later dissociated into single cells, transferred to six-well plates and incubated for 10 days. $\mathrm{n}=3$ independent experiments, with triplicate wells for each drug concentration. Data are presented as the mean \pm SEM. SI, survival index; FMCA, fluorometric microculture cytotoxicity assay; Conc, concentration; GFP, green fluorescent protein. 


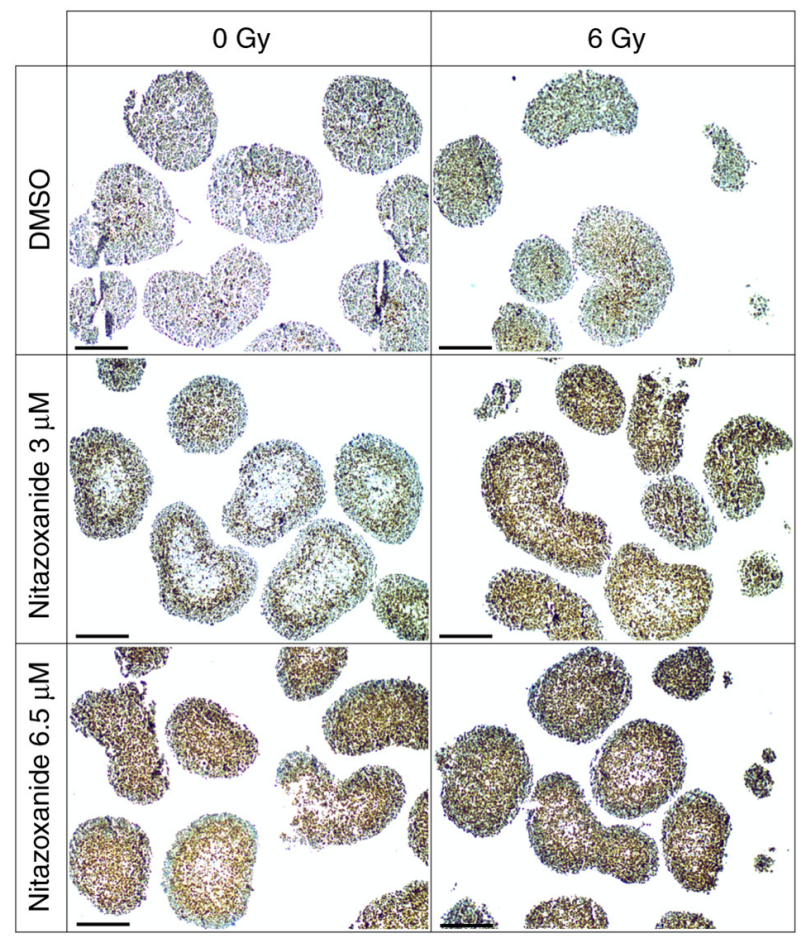

Figure 2. Immunohistochemistry with $\gamma-\mathrm{H} 2 \mathrm{AX}$. Immunohistochemical expression of $\gamma$-H2AX in HCT116 GFP cells cultured as spheroids for 7 days, then irradiated ( $6 \mathrm{~Gy})$ at $4-6 \mathrm{~h}$ after addition of drug and harvested $24 \mathrm{~h}$ later. Spheroids were originally evaluated at $x 400$ magnification. The size of spheroids were typically 400-500 $\mu \mathrm{m}$. Scale bar, $200 \mu \mathrm{m}$. GFP, green fluorescent protein; $\gamma-\mathrm{H} 2 \mathrm{AX}, \gamma-\mathrm{H} 2 \mathrm{~A}$ histone family member $\mathrm{X}$.

the animals then gained weight with no obvious differences between the groups. Animal behaviour was seemingly normal and similar in all study groups. The maximum size observed for a single tumor during the present study was length $2.30 \mathrm{~cm}$ and width $1.60 \mathrm{~cm}$, corresponding to a volume of $2.59 \mathrm{~cm}^{3}$ and was observed at day 28 in the control vehicle group.

The TVs increased in all groups throughout the study and the vehicle-treated animals developed the largest tumours (Fig. 3A). Animals treated with nitazoxanide and/or radiation developed smaller tumours compared with those treated with the vehicle. Moreover, the combination treatment of nitazoxanide + radiation resulted in no further inhibition of tumour growth compared with radiation only.

Histopathological examination of tumour xenografts. The nitazoxanide + radiation group was the only group with a TCP significantly lower than that of the control (Fig. 3B; Table III); however, there was also a strong trend towards lower TCP in the radiation only group, compared with control (Fig. 3B; Table III). Both groups treated with radiation $+/$ - nitazoxanide developed a TCV that was significantly lower than that of the control (Fig. 3C; Table III). Furthermore, there was a strong trend towards a lower TCV in the nitazoxanide only group compared with the control group (Fig. 3C; Table III). Visual inspection results indicated that the tumour xenografts typically consisted of an outer rim of viable and more centrally located pyknotic HCT116 GFP cells and necrosis, and a lesser degree of mouse tissue (stroma, fat, blood vessels and immune cells) (Fig. 3D).
Table III. TCP and TCV.

\begin{tabular}{lc} 
A, TCP & \\
\hline Treatment & P-value \\
\hline Control (vehicle) vs. control (vehicle) +6 Gy & 0.0587 \\
Control (vehicle) vs. nitazoxanide & 0.5880 \\
Control (vehicle) vs. nitazoxanide + 6 Gy & $0.0074^{\mathrm{a}}$ \\
Control (vehicle) + 6 Gy vs. nitazoxanide & 0.5303 \\
Control (vehicle) + 6 Gy vs. nitazoxanide +6 Gy & 0.8631 \\
Nitazoxanide vs. nitazoxanide + 6 Gy & 0.1503
\end{tabular}

B, TCV

Treatment

P-value

Control (vehicle) vs. control (vehicle) + 6 Gy

$0.0045^{\mathrm{a}}$

Control (vehicle) vs. nitazoxanide

0.0529

Control (vehicle) vs. nitazoxanide $+6 \mathrm{~Gy}$

$0.0019^{\mathrm{a}}$

Control (vehicle) +6 Gy vs. nitazoxanide

0.7579

Control (vehicle) +6 Gy vs. nitazoxanide +6 Gy

0.9956

Nitazoxanide vs. nitazoxanide +6 Gy

0.6047

${ }^{\text {a }}<0.01$. Differences in TCP and TCV were calculated using one-way ANOVA followed by Tukey's multiple comparisons test. Three animals that were euthanized pre-term were excluded from the analysis. $\mathrm{P}<0.05$ was used to indicate statistical significance in all experiments. $\mathrm{TCP}$, tumor cell percentage; TCV, tumor cell volume.

\section{Discussion}

Nitazoxanide, formally known as 2-(acetyloxy)-N-(5-nitro-2thiazolyl) benz-amide NTZ, is a thiazolide compound that was discovered in 1984 and originally developed as a veterinary anthelminthic (18-20). It is a non-toxic and well-tolerated compound, with a broad anti-microbial activity, that was developed for human use in the 1990s and is the only anti-protozoal drug approved for use in children (21). Drug administration and pharmacokinetics of nitazoxanide are advantageous for use in humans. Thus, plasma concentrations above $6 \mu \mathrm{mol} / 1 \mathrm{can}$ be reached in humans after a single oral dose of $500 \mathrm{mg}$ and $\mathrm{C}_{\max }$ in plasma is reached at 2-6 h after administration $(12,22)$.

The potential drug-repurposing of nitazoxanide into an anticancer drug could bypass early stages of drug development, and offers a faster and cheaper drug development process compared to with de novo anticancer drug discovery (23). Moreover, oral administration of nitazoxanide offers many numerous economic advantages compared with standard intravenous administrated chemotherapy since it requires less personnel and equipment.

Although initially designed as an anti-microbial drug, the preclinical antitumour activities of nitazoxanide in vitro and in vivo have been observed over the last decade $(12,18,19,21,24,25)$. The reported preclinical anti-cancer properties are promising for the development of nitazoxanide into a novel chemotherapeutic compound. These properties involve crucial antitumoural metabolic and 
A Nitazoxanide in HCT116 GFP xenograft tumors

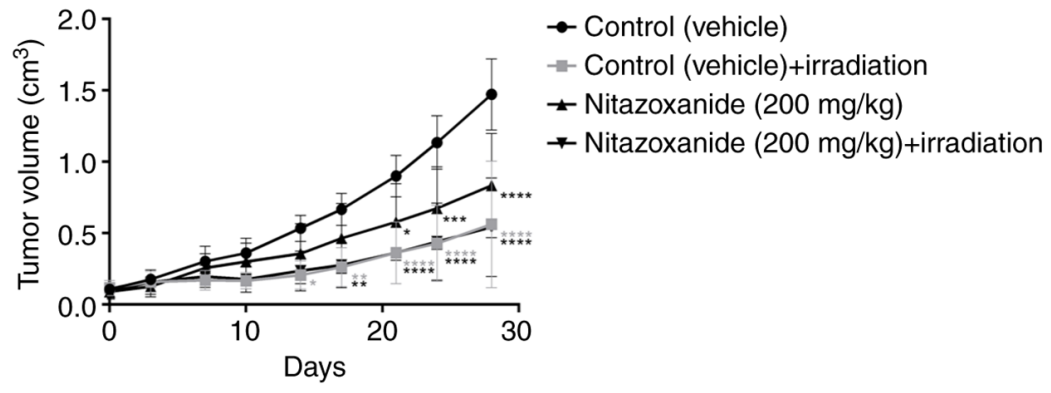

B Fraction of HCT116 GFP cells in the tumor

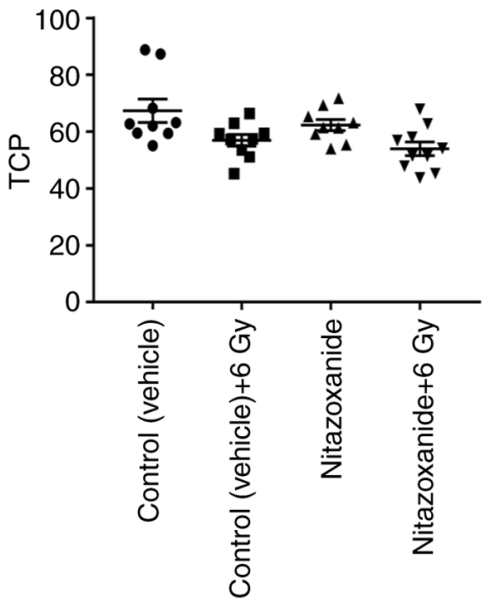

C HCT116 GFP mass of the tumor $\left(\mathrm{cm}^{3}\right)$

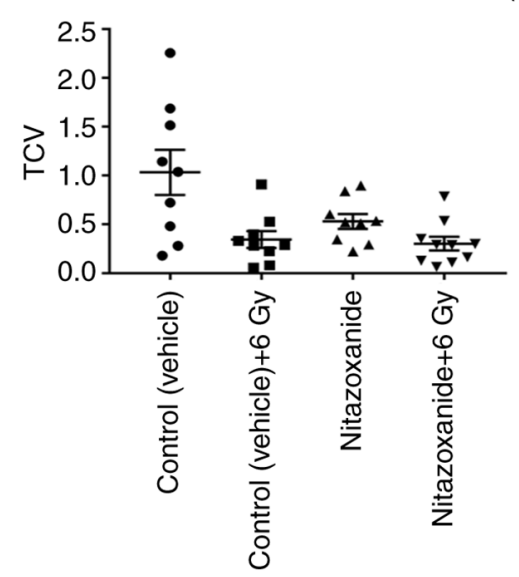

$\mathrm{D}$

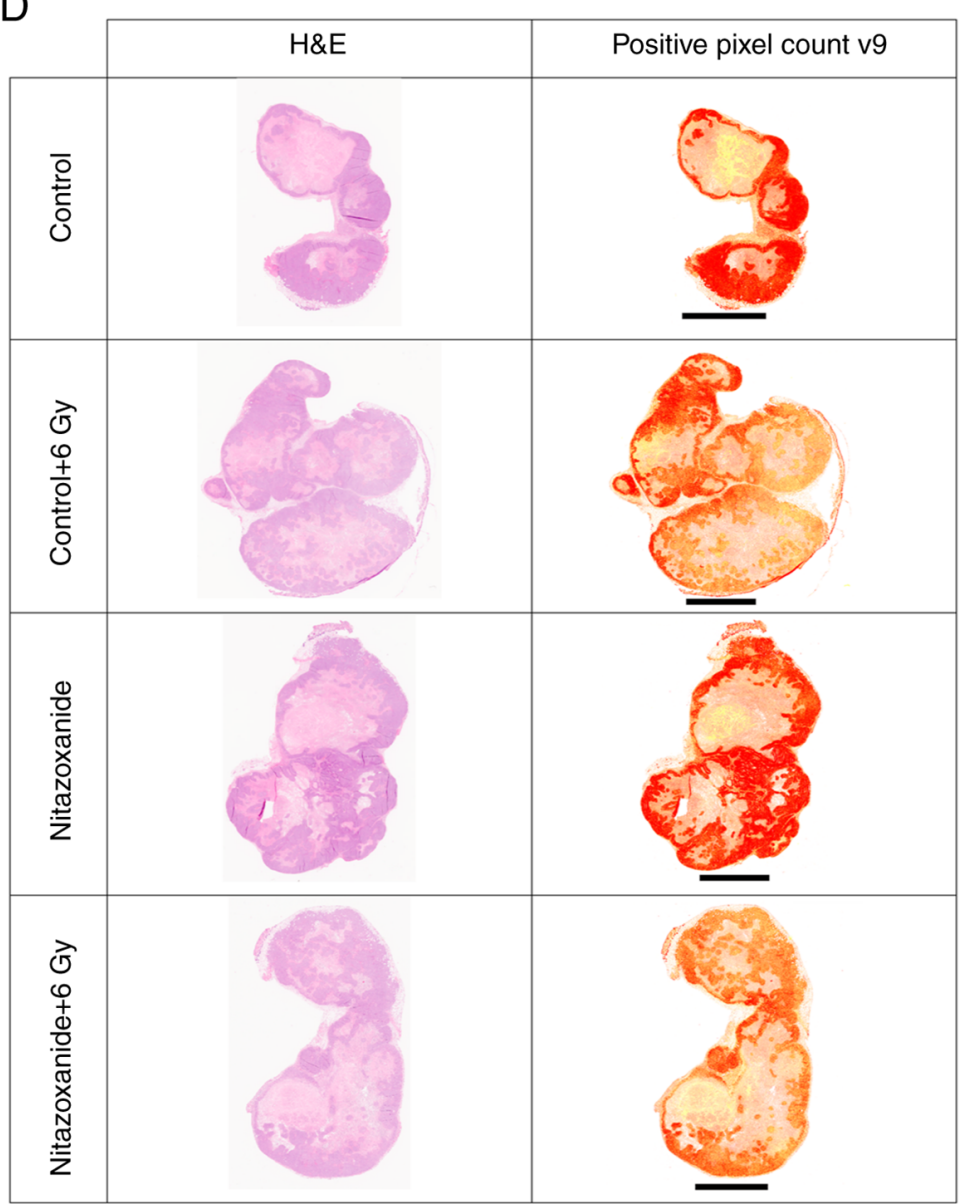

Figure 3. HCT116 GFP cell xenograft tumours in mice. (A) At day 0, drug/vehicle administration started twice daily for 3 days (only one administration was given on day 2). Vehicle control (1\% CMC in PBS with $8 \%$ DMSO) and nitazoxanide ( $200 \mathrm{mg} / \mathrm{kg}$ ) were administered via oral gavage. At 3-4 h after the last administration, the animal tumours were irradiated with $6 \mathrm{~Gy}$. ${ }^{*} \mathrm{P}<0.05,{ }^{* * *} \mathrm{P}<0.01,{ }^{* * * *} \mathrm{P}<0.001,{ }^{* * * * * *} \mathrm{P}<0.0001$ vs. vehicle control. HCT116 GFP cell xenograft tumours were embedded in paraffin, sectioned, evaluated for H\&E staining and scanned. (B) TCP was calculated with the analysis algorithm 'Positive Pixel Count v9' in Aperio ImageScope (v12.3.2.8013). (C) TCV was calculated to evaluate the HCT116 GFP cell mass of the tumour. (D) Typical examples from the four treatment groups (control, control $+6 \mathrm{~Gy}$, nitazoxanide and nitazoxanide $+6 \mathrm{~Gy}$ ) are shown. Please note that the size of tumour in this figure is dependent on level of sectioning and does not necessarily correlate to tumour volume. Scale bar, $4 \mathrm{~mm}$. CMC, carboxymethylcellulose; GFP, green fluorescent protein; $\mathrm{TCV}$, tumour cell volume; TCP, tumour cell percentage.

pro-death signalling, such as drug detoxification, unfolded protein response, autophagy, immunological responses and interference in cell signalling pathways, such as the MAPK pathway via interference with glutathione $S$-transferase $\pi 1$ or c-Myc $(18,19,21)$.
Our previous study described nitazoxanide as a hit-compound in a screening process designed to identify drugs with selective activity against quiescent and glucose-deprived tumour cells, and exposure of colon cancer cells to nitazoxanide resulted in OXPHOS-inhibition 
and reduced hypoxia in spheroids (12). Moreover, the nitro group in the nitrothiazole moiety can be converted into a free radical $(21,26)$. These properties could potentially be of substantial value in the development of a novel radiosensitizer, since the major effect of radiation is via the formation of free oxygen radicals, leading to DSBs.

In line with our previous observations, the present results demonstrated that nitazoxanide showed selective activity against spheroids compared with monolayers, as determined in the total cell kill assay. The seemingly protective effect of radiation to nitazoxanide in the monolayer experiments incubated for 7, but not 3 days was unexpected, and is suggested to be explained by the selective effect of nitazoxanide against the quiescent and glucose-deprived cells in wells not irradiated (12), due to faster cell proliferation in these wells secondary to lack of inhibitory radiation. The non-irradiated cells exposed to nitazoxanide will be confluent and glucose-deprived after, but not before, 3 days, compared with irradiated cells. This would explain the higher effect of nitazoxanide after 7 days, compared with 3 days, in non-irradiated cells, and the transient radiation effect would explain the higher cell survival after 7 days compared with 3 days in irradiated wells. This was in agreement with nitazoxanide being more active against cells in medium with low glucose and $\mathrm{pH}$ (12), and is theoretically advantageous since lower toxicity toward normal cells, exposed to normal glucose levels and $\mathrm{pH}$, is expected.

No synergistic interaction between nitazoxanide and radiation could be observed for monolayer or spheroid cultured cells in the total cell kill assay. This assay is useful for viability measurements in high-throughput screening experiments but may miss effects secondary to cell cycle arrest and cell senescence, as well as those in small subpopulations of cells. Thus, the radiosensitizing property observed in the clonogenic assay on spheroids was in accordance with the synergistic cell inhibitory effect of nitazoxanide on clonogenic cells, rather than synergistic cytotoxicity among all cells.

The possible mechanism underlying the induction in DSBs in spheroids by nitazoxanide itself is beyond the scope of the present study, but was not unexpected since nitazoxanide can induce cell growth inhibition via the formation of free radicals $(21,26)$. Interestingly, the increase in DSBs in central parts of spheroids exposed to the combination of nitazoxanide and radiation compared with spheroids exposed to nitazoxanide or radiation only indicates a selective radiosensitizing effect towards more centrally located cells. This supports the hypothesis that reduced hypoxia and formation of free radicals are important mechanisms underlying the radiosensitization. However, a potential study limitation is the absence of experiments analyzing reoxygenation in spheroids during the present study. The radiosensitizing effect in spheroids could be observed in the clonogenic assay, but not in the total cell kill assay, and may be compatible with a low number of DSBs formed after exposure to the combination treatment that are only sufficient to inhibit the clonogenicity of tumour driving cells, but not enough to further induce direct cytotoxicity. Since clonogenic tumour-driving stem cells are considered to be located in quiescent and glucose-deprived parts of tumours, a selective synergistic interaction with radiation in this tumour compartment may be beneficial.

Overall, the current data support the hypothesis that nitazoxanide acts as a radiosensitizer in tumour-driving, quiescent parts of tumours. Theoretically, the combination of a radiosensitizer effective against high-proliferative tumour cells (e.g. 5-FU) (1) with a radiosensitizer that targets quiescent clonogenic tumour cells (e.g. nitazoxanide) could enhance the anticancer effect of radiation via both an increased bioequivalent radiation dose to the tumour and a reduced radiation effect in surrounding normal cells.

Enhancement of the radiation effect by nitazoxanide was not observed in vivo as determined via tumour size. However, given the synergistic interaction between nitazoxanide and radiation only in quiescent clonogenic cells, as suggested from the in vitro experiments, this finding was not surprising. In addition, the current study recognized that a major problem with examining the radiosensitization effects in the HCT116 GFP cell murine xenograft model was that the tumours only partially consist of tumour cells, alongside considerable parts with necrosis and mouse tissue. Therefore, it was important to evaluate the TCP and TCV in the xenografts. Notably, the only group that developed a significantly lower TCP compared with the control was the nitazoxanide + radiation group. This may indicate a selective radiosensitization effect of nitazoxanide against the tumour-driving HCT116 GFP cell mass of the tumour. However, no difference was observed compared with the radiation only group. As expected, although a strong trend towards a lower TCP compared with the control was identified in the radiation only group, the in vivo data suggests that this relatively high radiation dose also affects normal cells to a considerable extent.

Single treatment with nitazoxanide for only 3 days resulted in a statistically significant inhibition of tumour growth in vivo. A strong trend towards a smaller TCV compared with the control, in contrast to similar TCPs, is compatible with the selective effect of nitazoxanide against the tumour-driving HCT116 GFP cell mass of the tumour, but is more suggestive of an inhibitory effect on quiescent and glucose-deprived tumour compartments consisting of both HCT116 GFP cells and transformed normal cells. This was unexpected, since continuous treatment with nitazoxanide for 28 days in a similar xenograft model did not significantly inhibit tumour growth (12). However, the antitumour effect of single treatment with nitazoxanide indicates that the mechanisms important in vivo are complex. Drug doses, administration schedules, the build-up and wash-out of an active compound in the tumour, radiation dose and follow-up time are factors that may impact on the possibility to observe radiosensitization and single drug effects in vivo. Moreover, although spheroids are considered to better reflect solid tumours in vivo compared with monolayers, the situation in vivo is more complex and several factors, such as pharmacokinetics, interaction with other cell types (stroma, immune cells, fat) and elevated intratumor pressure, will affect anticancer drug and radiation therapy, and are difficult to mimic in vitro.

In conclusion, the current study demonstrated that nitazoxanide presented several characteristics that make it highly interesting for repurposing into an anticancer drug, as well as for use in combination with radiation. However, other models are required to reflect the potential of nitazoxanide as a radiosensitizer in vivo. Since nitazoxanide shows selective effects against quiescent and glucose-deprived tumour compartments and radiosensitization of clonogenic cells in 
these regions, in vivo experiments with serial orthotopic transplantation of tumours exposed to nitazoxanide and radiation may be needed to provide proof of principle evidence of the long term radiosensitization effects of this combination.

\section{Acknowledgements}

The laboratory work by Ms Lena Lenhammar, our late Ms Christina Leek and Dr Sharmineh Mansoori at the Department of Medical Sciences, Uppsala University Hospital (Uppsala, Sweden) is gratefully acknowledged. Adlego Biomedical AB (Astrid Fagreaus Laboratoriet, Solna, Sweden) carried out the tumour xenograft experiments in mice.

\section{Funding}

This work was supported by grants from Swedish Cancer Society (grant no. 462430020), Swedish Foundation for Strategic Research (grant no. 2008-03000) and Lions Cancer Research Fund.

\section{Availability of data and materials}

The datasets used and/or analysed during the current study are available from the corresponding author on reasonable request.

\section{Authors' contributions}

HK and PN designed the experiments, wrote the paper and are responsible for confirming the authenticity of the raw data. MF and RL helped design the experiments. WS helped design the spheroid experiments. The laboratory work was performed by HK. All authors read and approved the final manuscript.

\section{Ethics approval and consent to participate}

Mouse experiments were approved by the Regional Animal Experimental Ethics Committee in Stockholm (North; approval nos. N37/15 and N188/15).

\section{Patient consent for publication}

Not applicable.

\section{Competing interests}

Uppsala University has received an unconditional grant from Romark LC for studies of nitazoxanide. This grant has not supported any part of the experiments described in this manuscript.

\section{References}

1. Shewach DS and Lawrence TS: Antimetabolite radiosensitizers J Clin Oncol 25: 4043-4050, 2007.

2. Lawrence TS, Blackstock AW and McGinn C: The mechanism of action of radiosensitization of conventional chemotherapeutic agents. Semin Radiat Oncol 13: 13-21, 2003.

3. Dai T and Shah MA: Chemoradiation in oesophageal cancer. Best Pract Res Clin Gastroenterol 29: 193-209, 2015.
4. Spithoff K, Cummings B, Jonker D and Biagi JJ; Gastrointestinal Cancer Disease Site Group: Chemoradiotherapy for squamous cell cancer of the anal canal: A systematic review. Clin Oncol (R Coll Radiol) 26: 473-487, 2014.

5. Morris ZS and Harari PM: Interaction of radiation therapy with molecular targeted agents. J Clin Oncol 32: 2886-2893, 2014.

6. Koning CC, Wouterse SJ, Daams JG, Uitterhoeve LL, van den Heuvel MM and Belderbos JS: Toxicity of concurrent radiochemotherapy for locally advanced non-small-cell lung cancer: A systematic review of the literature. Clin Lung Cancer 14: 481-487, 2013.

7. DeVita VT, Lawrence TS and Rosenberg SA: Cancer Principles and Practice of Oncology. Wolters Kluwer, Philadelphia, PH, pp280, 2015.

8. Ivashkevich A, Redon CE, Nakamura AJ, Martin RF and Martin OA: Use of the $\gamma-\mathrm{H} 2 \mathrm{AX}$ assay to monitor DNA damage and repair in translational cancer research. Cancer Lett 327: 123-133, 2012.

9. Radford IR: The level of induced DNA double-strand breakage correlates with cell killing after X-irradiation. Int J Radiat Biol Relat Stud Phys Chem Med 48: 45-54, 1985.

10. Brown JM: The hypoxic cell: A target for selective cancer therapy-eighteenth Bruce F. Cain Memorial Award lecture. Cancer Res 59: 5863-5870, 1999.

11. Bernier J, Hall EJ and Giaccia A: Radiation oncology: A century of achievements. Nat Rev Cancer 4: 737-747, 2004.

12. Senkowski W, Zhang X, Olofsson MH, Isacson R, Höglund U, Gustafsson M, Nygren P, Linder S, Larsson R and Fryknäs M: Three-Dimensional cell culture-based screening identifies the anthelmintic drug nitazoxanide as a candidate for treatment of colorectal cancer. Mol Cancer Ther 14: 1504-1516, 2015.

13. Overgaard J: Hypoxic radiosensitization: Adored and ignored. J Clin Oncol 25: 4066-4074, 2007.

14. DeVita VT Jr and Chu E: A history of cancer chemotherapy. Cancer Res 68: 8643-8653, 2008.

15. Lindhagen E, Nygren $P$ and Larsson R: The fluorometric microculture cytotoxicity assay. Nat Protoc 3: 1364-1369, 2008.

16. Franken NA, Rodermond HM, Stap J, Haveman J and van Bree C: Clonogenic assay of cells in vitro. Nat Protoc 1: 2315-2319, 2006.

17. Yeh PJ, Hegreness MJ, Aiden AP and Kishony R: Drug interactions and the evolution of antibiotic resistance. Nat Rev Microbiol 7: 460-466, 2009.

18. Di Santo N and Ehrisman J: A functional perspective of nitazoxanide as a potential anticancer drug. Mutat Res 768: 16-21, 2014.

19. Muller J, Sidler D, Nachbur U, Wastling J, Brunner T and Hemphill A: Thiazolides inhibit growth and induce glutathione-S-transferase Pi (GSTP1)-dependent cell death in human colon cancer cells. Int J Cancer 123: 1797-1806, 2008.

20. Rossignol JF and Maisonneuve H: Nitazoxanide in the treatment of Taenia saginata and Hymenolepis nana infections. Am J Trop Med Hyg 33: 511-512, 1984.

21. Fan-Minogue H, Bodapati S, Solow-Cordero D, Fan A, Paulmurugan R, Massoud TF, Felsher DW and Gambhir SS: A c-Myc activation sensor-based high-throughput drug screening identifies an antineoplastic effect of nitazoxanide. Mol Cancer Ther 12: 1896-1905, 2013.

22. Stockis A, Deroubaix X, Lins R, Jeanbaptiste B, Calderon P and Rossignol JF: Pharmacokinetics of nitazoxanide after single oral dose administration in 6 healthy volunteers. Int J Clin Pharmacol Ther 34: 349-351, 1996.

23. Wurth R, Thellung S, Bajetto A, Mazzanti M, Florio T and Barbieri F: Drug-repositioning opportunities for cancer therapy: Novel molecular targets for known compounds. Drug Discov Today 21: 190-199, 2016.

24. Di Santo N and Ehrisman J: Research perspective: Potential role of nitazoxanide in ovarian cancer treatment. Old drug, new purpose? Cancers (Basel) 5: 1163-1176, 2013.

25. Brockmann A, Strittmatter T, May S, Stemmer K, Marx A and Brunner T: Structure-function relationship of thiazolide-induced apoptosis in colorectal tumor cells. ACS Chem Biol 9: 1520-1527, 2014.

26. Hemphill A, Mueller $\mathrm{J}$ and Esposito M: Nitazoxanide, a broad-spectrum thiazolide anti-infective agent for the treatment of gastrointestinal infections. Expert Opin Pharmacother 7: 953-964, 2006

c) (i) $\Theta$ This work is licensed under a Creative Commons

cc) Attribution-NonCommercial-NoDerivatives 4.0 International (CC BY-NC-ND 4.0) License. 